D.O.I.: $10.3895 / \mathrm{S} 1808-04482007000100006$

\title{
ESTRATÉGIA DE PRODUÇÃO, MELHORES PRÁTICAS E MEDIÇÃO DE DESEMPENHO: REVISÃO, LACUNAS E PLANEJAMENTO PARA FUTURAS PESQUISAS
}

\section{MANUFACTURING STRATEGY, BEST PRACTICES AND PERFORMANCE MEASUREMENT: REVISION, RESEARCH GAPS AND AN AGENDA FOR FUTURE STUDIES}

\author{
Eliciane Maria da Silva ${ }^{1}$; Fernando César Almada Santos ${ }^{2}$ \\ ${ }^{1}$ Universidade de São Paulo - USP - São Carlos - Brasil eliciane@sc.usp.br \\ ${ }^{2}$ Universidade de São Paulo - USP - São Carlos - Brasil almada@sc.usp.br
}

\begin{abstract}
Resumo
Este artigo tem o objetivo principal de identificar lacunas de pesquisa e recomendar um planejamento para futuros trabalhos na área de estratégia de produção, sobretudo, aqueles que correlacionam melhores práticas e o desempenho operacional. Por se tratar de um tema abrangente, que é discutido há quase meio século, esta pesquisa contribui também para mostrar as correntes de pesquisas existentes a partir de uma nova visão global e sistematização da literatura. Realiza-se, então, uma completa revisão bibliográfica em torno da estratégia de produção. Das seis correntes de pesquisas identificadas, quatro estão em andamento. $O$ artigo finaliza com uma reflexão sobre as lacunas encontradas e sugere futuros trabalhos.
\end{abstract}

Palavras-chave: estratégia de produção, melhores práticas, desempenho operacional.

\section{Introdução}

A estratégia de produção é um campo de pesquisa bem estabelecido que se originou, há quase meio século, nos trabalhos clássicos de Skinner (1969); Hayes e Wheelwright (1984); Fine e Hax (1985) e Hill (1993). Contudo a sua prática é recente e de extrema importância nas organizações que buscam um posicionamento competitivo.

No ambiente acadêmico, periódicos de renomes exibem trabalhos tradicionais na área como o "International Journal of Operations \& Production Management" que teve artigos reeditados em 2005 e a revista "Gestão \& Produção" que incluiu em 2005 uma edição especial sobre estratégia de produção. 
Dada a amplitude deste tema, o presente trabalho tem como objetivo principal refletir sobre as lacunas encontradas no referencial teórico e sugerir um planejamento para futuros estudos na área de estratégia de produção, principalmente, aqueles que correlacionam melhores práticas e o desempenho operacional. Mediante a apresentação de um novo construto que representa uma visão global da estratégia de produção e subdividindo-a em seis correntes de pesquisa, a próxima seção apresenta uma completa revisão bibliográfica e sistematização da literatura. Na seqüência são identificadas as lacunas de pesquisas e recomendadas propostas para futuros trabalhos. Por fim, são expostas as conclusões e as bibliografias utilizadas.

\section{Estratégia de produção, melhores práticas e medição de desempenho}

Uma maneira compreensível de abordar a estratégia de produção é subdividí-la em seu conteúdo e processo de elaboração. O conteúdo da estratégia de produção diz respeito às prioridades competitivas, áreas de decisões, melhores práticas de produção e medição de desempenho (SWAMIDASS e NEWELL, 1987; ADAM e SWAMIDASS, 1989). Já o processo se refere às metodologias seguidas para o desenvolvimento da estratégia de produção (SWAMIDASS e NEWELL, 1987). De forma complementar, Krause, Pagell e Curkovic (2001) afirmam que o foco do conteúdo se preocupa com o que a organização almeja competir e o processo centraliza em como a estratégia de produção é desenvolvida. A Figura 1 ilustra um novo construto criado pelos autores que apresenta seis linhas de pesquisa baseadas na revisão bibliográfica e sua explicação é descrita a seguir.

Figura 1 - Um novo construto dos fundamentos da estratégia de produção 


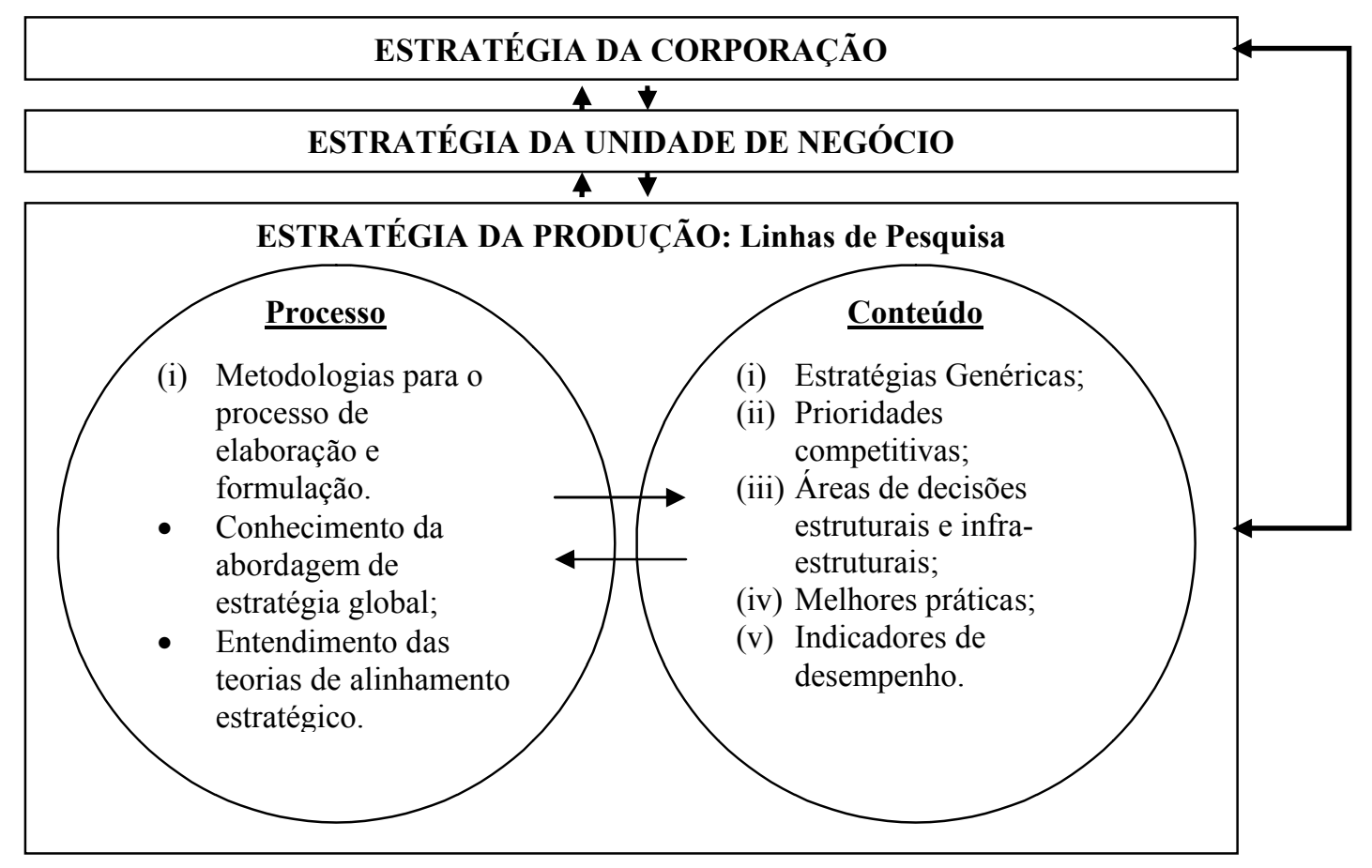

Na Figura 1 as duas perspectivas (conteúdo e processo) estão fortemente inter-relacionadas. O processo deve estar apto para administrar o conteúdo. Além disso, observa-se que sem o processo o conteúdo não se torna efetivo, por outro lado, se não houver conteúdo o processo será apenas um método incapaz de realizar qualquer coisa na organização. Ao escolher uma metodologia para a formulação das estratégias de produção, é necessário ter total domínio sobre a abordagem de elaboração das estratégias globais e entender as teorias do alinhamento estratégico. Ambas são compreendidas como teorias mais amplas que não estão somente no âmbito da estratégia de produção e por esta razão aparecem com marcadores na Figura 1.

A gestão da produção, com base nas diretrizes da estratégia corporativa, desenvolve ou adapta uma metodologia para elaborar suas estratégias. Duas abordagens são as mais comuns para o desenvolvimento da estratégia corporativa - estratégias competitivas de Porter (1980) e a Visão Baseada em Recursos (RBV) que foi impulsionada por Wernerfelt (1984); Barney (1991) e Grant (1991). Porter (1980), por meio de uma visão top-down, introduz que o processo de formulação das estratégias globais inicia-se pela análise do ambiente mediante cinco forças competitivas competidores, fornecedores, clientes, novos entrantes e substitutos. Posteriormente a análise do ambiente, a empresa posiciona-se no mercado a partir da escolha de uma das três estratégias competitivas - diferenciação, liderança no custo e enfoque. De forma oposta, em uma visão bottomup, Wernerfelt (1984) defende que a empresa pode desenvolver suas estratégias fundamentadas em seus recursos. Estes podem ser utilizados para desenvolver diferentes produtos, sendo assim, possível encontrar um posicionamento de mercado ótimo e obter vantagem competitiva sobre os concorrentes. 
Hayes e Wheelwright (1984), nesta mesma linha de pensamento, afirmam que a manufatura desempenha papéis que podem ser vistos como quatro estágios graduais de desenvolvimento ao longo de um espaço de tempo. Em um extremo (no primeiro estágio) a função produção pode oferecer pequena contribuição para sucesso da companhia, em outro âmbito (no quarto estágio), ela é provedora de maior fonte de vantagem competitiva. Dada a importância da função produção na organização, alguns pesquisadores - Hörte, Lindberg e Tunälv (1987); Platts (1994); Schroeder (1993); Hill (1993) e Mills, Platts e Gregory (1995) - contribuem para desenvolverem metodologias para elaboração de estratégias e outros - Semler, 1997 e Stepanovich e Mueller, 2002 - sustentam a teoria do alinhamento estratégico, sendo esta fundamental para criar um trabalho de alto desempenho a partir da busca de maior eficiência e efetividade coletiva e individual na empresa.

Em outro âmbito, sob a perspectiva do conteúdo da estratégia de produção (Figura 1), a função produção, em concordância com a estratégia de negócio e da corporação, estabelece estratégias genéricas e institui prioridades competitivas. Na seqüência, é preciso decidir sobre os investimentos em questões estruturais, infra-estruturais e práticas de produção. Então, para que se avaliem os resultados é essencial a criação de indicadores de desempenhos. Esses por sua vez medirão a performance da manufatura e o seus resultados poderão estimular a competitividade da organização como um todo.

A abordagem sobre estratégia genérica da produção foi proposta por Kotha e Orne (1989), cujo trabalho envolveu a nomeação de oito tipologias de estratégias genéricas baseadas em três dimensões. Contudo, um estudo mais completo é realizado por Bozarth e Mcdermoth (1998) que elaboram uma classificação para as estratégias genéricas de produção.

Já as prioridades competitivas tornaram-se relevantes após o trabalho de Skinner (1969) no qual descreve alguns padrões comuns para mensurar o desempenho da manufatura: ciclos menores de entregas do produto, produto com qualidade e confiabilidade, cumprimento com a promessa de entrega, habilidade para produzir novos produtos rapidamente, flexibilidade para ajustar mudanças no volume e custos baixos. Dando seqüência e complementando o trabalho de Skinner (1969) novas categorias de prioridade competitivas foram definidas por outros pesquisadores: Schmenner (apud Vickery, Droge e Markland, 1997); Hayes e Wheelwright (1984); Swamidass e Newel, 1987; Hörte, Lindberg e Tunälv, 1987; Gerwin, 1987; Adam e Swamidass, 1989; Leong, Snyder e Ward, 1990; Garvin, 1993; Schroeder, 1993; Hill, 1993; Stonebraker e Leong, 1994; Pires, 1995; Krajewski e Ritzman, 2000; Santos, 2000; Jiménez e Lorente, 2001; Slack et al., 2002; Vachon e Klassen, 2006. Nestes estudos as prioridades competitivas de maior inserção são qualidade, rapidez, flexibilidade e custo.

Skinner (1969) também inseriu cinco áreas de decisões da produção - planta e equipamento, planejamento e controle da produção, mão-de-obra; desenvolvimento de produto e engenharia, 
organização e gerenciamento - nas quais gerentes devem analisar e selecionar alternativas de decisões que apóiem a estratégia corporativa. Dentre os diferentes trabalhos que colaboraram para o entendimento sobre a importância das áreas de decisões da produção estão os de: Skinner (1969); Hayes e Wheelwright (1984); Schroeder, Anderson e Cleveland (1986); Hörte, Lindberg e Tunälv (1987); Stonebraker e Leong (1994); Paiva, Carvalho Júnior e Fensterseifer (2004).

Mais recentemente, as melhores práticas da manufatura são incluídas ao conteúdo da estratégia de produção. Adam e Swamidass (1989) identificam que temas como just-in-time (JIT), produtividade e qualidade não estão integrados com a manufatura e à estratégia de produção. Mills, Platts e Gregory (1995) enfatizam que uma visão mais ampla a respeito da estratégia de produção é representada pela integração das melhores práticas com as áreas de decisões da manufatura. Voss (1995), na mesma época, já considera as melhores práticas como um dos paradigmas da estratégia de produção.

Freqüentemente pesquisadores correlacionam melhores práticas com medição de desempenho operacional, sendo que a maioria dos indicadores de desempenho é relacionada as quatro prioridades competitivas mais citadas na literatura: qualidade, rapidez, flexibilidade e custo. A Tabela 1 apresenta um resumo de trabalhos empíricos que correlacionam melhores práticas e medição de desempenho em algumas áreas de decisões da estratégia de produção.

Tabela 1 - Trabalhos empíricos que correlacionam melhores práticas e medição de desempenho

\begin{tabular}{ccc}
\hline Áreas & Autores & \multicolumn{1}{c}{ Práticas estudadas (P) e Indicadores de Desempenho (ID) } \\
\hline $\begin{array}{c}\text { Tecno- } \\
\text { logia }\end{array}$ & Boyer (1998) & $\begin{array}{c}\text { P: automação baseada em desenvolvimento de projeto, sistemas de manufatura } \\
\text { e sistemas administrativos. } \\
\text { ID: relacionados à custo, entrega, qualidade e flexibilidade. }\end{array}$ \\
\hline $\begin{array}{c}\text { Integra- } \\
\text { ção } \\
\text { vertical }\end{array}$ & $\begin{array}{c}\text { Gilley e Rasheed } \\
(2000)\end{array}$ & $\begin{array}{c}\text { P: "outsourcing periférico" e "outsourcing de atividades chaves (core)"; } \\
\text { ID: relacionados às estratégias de liderança no custo, diferenciação de mercado } \\
\text { e diferenciação em inovação. }\end{array}$ \\
\hline $\begin{array}{c}\text { Forne- } \\
\text { cedores }\end{array}$ & $\begin{array}{c}\text { Fynes, Voss e } \\
\text { Burca (2005) }\end{array}$ & $\begin{array}{c}\text { P: comunicação, comprometimento, adaptação, cooperação; } \\
\text { ID: indicadores de qualidade, desempenho nas entregas, custo e flexibilidade. }\end{array}$ \\
$\begin{array}{c}\text { Recur- } \\
\text { sos }\end{array}$ & $\begin{array}{c}\text { Banker et al. } \\
(1996)\end{array}$ & $\begin{array}{c}\text { P: times de trabalho de alto desempenho; } \\
\text { ID: indicadores de qualidade e produtividade. }\end{array}$ \\
\cline { 2 - 3 } humanos & $\begin{array}{c}\text { Youndt et al. } \\
(1996)\end{array}$ & $\begin{array}{l}\text { P: práticas de staff; de treinamento e de avaliação de desempenho. } \\
\text { ID: indicadores de qualidade, flexibilidade, escopo de flexibilidade e custo }\end{array}$ \\
\hline
\end{tabular}

Outros estudos atuais (SHAH e WARD, 2003; SWINK, NARASIMHAN e KIM, 2005; LAUGEN, BOER e FRICK, 2005; BEAUMONT, 2005) também analisam a influência das práticas de manufatura sob o desempenho operacional que são relacionadas às demais áreas da estratégia de produção - JIT, produção enxuta, capacidade da manufatura, entre outras.

Diante do referencial teórico apresentado até o momento torna-se possível refletir sobre as lacunas de pesquisas e sugerir propostas para futuros trabalhos. A próxima seção discute ambos os assuntos. 


\section{Estratégia de produção, melhores práticas e medição de desempenho}

A despeito da estratégia de produção ser debatida há quase meio século, o debate em algumas linhas de pesquisa ainda não se concretizou. Das seis correntes apresentadas na Figura 1, quatro estão em andamento: (a) as metodologias para o processo de formulação das estratégias, principalmente, no que diz respeito ao entendimento do alinhamento estratégico; (b) as prioridades competitivas; (c) as melhores práticas; e (d) os indicadores de desempenho.

Partindo para a linha de pesquisa sobre metodologias de formação das estratégias de produção, Sun e Hong (2002) observam a ausência de ferramentas ou métodos para uma perspectiva multifuncional no processo de elaboração da estratégia.

No âmbito do alinhamento estratégico, observa-se que na prática das organizações, esta linha de estudo ainda não está bem esclarecida. A saber, Nogueira, Alves Filho e Torkomian (2001) identificam possíveis ações combinadas entre as estratégias corporativas e de produção no setor de cerâmica. Os autores encontram falhas no alinhamento das estratégias e sugerem melhorias em práticas relacionadas às áreas de produção. Outros estudos que corroboram este problema são os de Silva e Santos (2005) e de Maia, Cerra e Alves Filho (2005). Não obstante, Paiva, Carvalho Júnior e Fenstersifer (2004) propõem um modelo, que busca uma maior coerência interna entre as categorias de decisões da estratégia de produção e da estratégia de negócio, nomeando-o de Rede de Valor de Operações (RVO). Mais tarde, Kippel, Antunes Júnior e Paiva (2005) colocam este modelo em um estudo empírico centrado em uma empresa rodoferroviária. Os autores contribuem para sanar o problema do alinhamento estratégico enfatizando a necessidade de adaptação do modelo utilizado para o setor de estudo específico.

Na perspectiva sobre o conteúdo das prioridades competitivas de produção, novas pesquisas contribuem para a sua evolução. Angell e Klassen (1999), após uma ampla revisão da literatura internacional, afirmam que a questão ambiental atende os requisitos para ser considerada uma prioridade competitiva da manufatura, mas reconhecem que essa perspectiva demanda um aprofundamento reflexivo, bem como novas abordagens empíricas. Posteriormente, Jiménez e Lorente (2001) e Vachon e Klassen (2006) apresentam argumentos que transformam a dimensão ambiental em uma nova prioridade competitiva da função produção. Todavia, faz necessária a realização de tais estudos no Brasil, uma vez que são raros, quando existentes, os trabalhos que integram a dimensão ambiental no contexto da estratégia de produção. Sugerem-se também, estudos a respeito de trade-offs entre as prioridades competitivas tradicionais (custo, qualidade, flexibilidade e desempenho nas entregas) incluindo as emergentes prioridades competitivas como a dimensão ambiental. 
Em se tratando do conteúdo das melhores práticas de produção, a literatura pesquisada assevera que há poucos estudos que investigam a integração das melhores práticas com o contexto específico da empresa, assim, algumas práticas são relevantes em pontos particulares do desenvolvimento das organizações podendo não ser aplicadas em outras (FITZ-ENZ, 1997; DAVIES e KOCHHAR, 2002; UNGAN, 2004, LAUGEN, BOER e FRICK, 2005). Esses autores ainda identificam a necessidade de investigar se as melhores práticas são as causas de desempenhos superiores nas organizações. Pilkington (1998) acrescenta outra falha a respeito da medição de desempenho ao dizer que alguns modelos conceituais que exploram a integração da estratégia de produção às estratégias de negócios e performance organizacional são específicos, pouco detalhados e genéricos. Neste sentido, a execução de mais estudos - integrando práticas, estratégia de produção, estratégia de negócio e desempenho - são recomendados para futuros trabalhos. Sugerem-se também, que estudos que correlacionam estratégias competitivas e de produção, especialmente, aqueles que avaliam a influência da adoção de práticas de produção sob o desempenho operacional e de negócio, utilizem (por meio de ajustes teóricos) definições específicas para o setor estudado. Boyer e Pagell (2000) constatam que não se devem utilizar mensurações gerais para estudos que analisam indústrias específicas. Vickery, Droge e Markland (1993) em um estudo voltado para o setor de móveis desenvolvem definições apropriadas de qualidade.

Outros autores ainda acrescentam que as práticas pesquisadas são extremamente específicas resultando em uma comparação limitada entre os estudos (Bolden et al., 1997). Estudos que avaliam programas que contém práticas em comuns ou a utilização de práticas progressivas, já têm sido efetuados por pesquisadores como Christiansen et al. (2003); Narasimhan, Swink e Kim (2005) e Cua, Mckone e Schroeder (2001). Porém, a literatura até o momento se mostra carente de mais estudos que envolvam este assunto.

A Figura 2 ilustra as correntes de pesquisas em andamento e apresenta uma síntese de um planejamento para futuros trabalhos.

Figura 2 - Planejamento para futuros trabalhos em estratégia de produção 

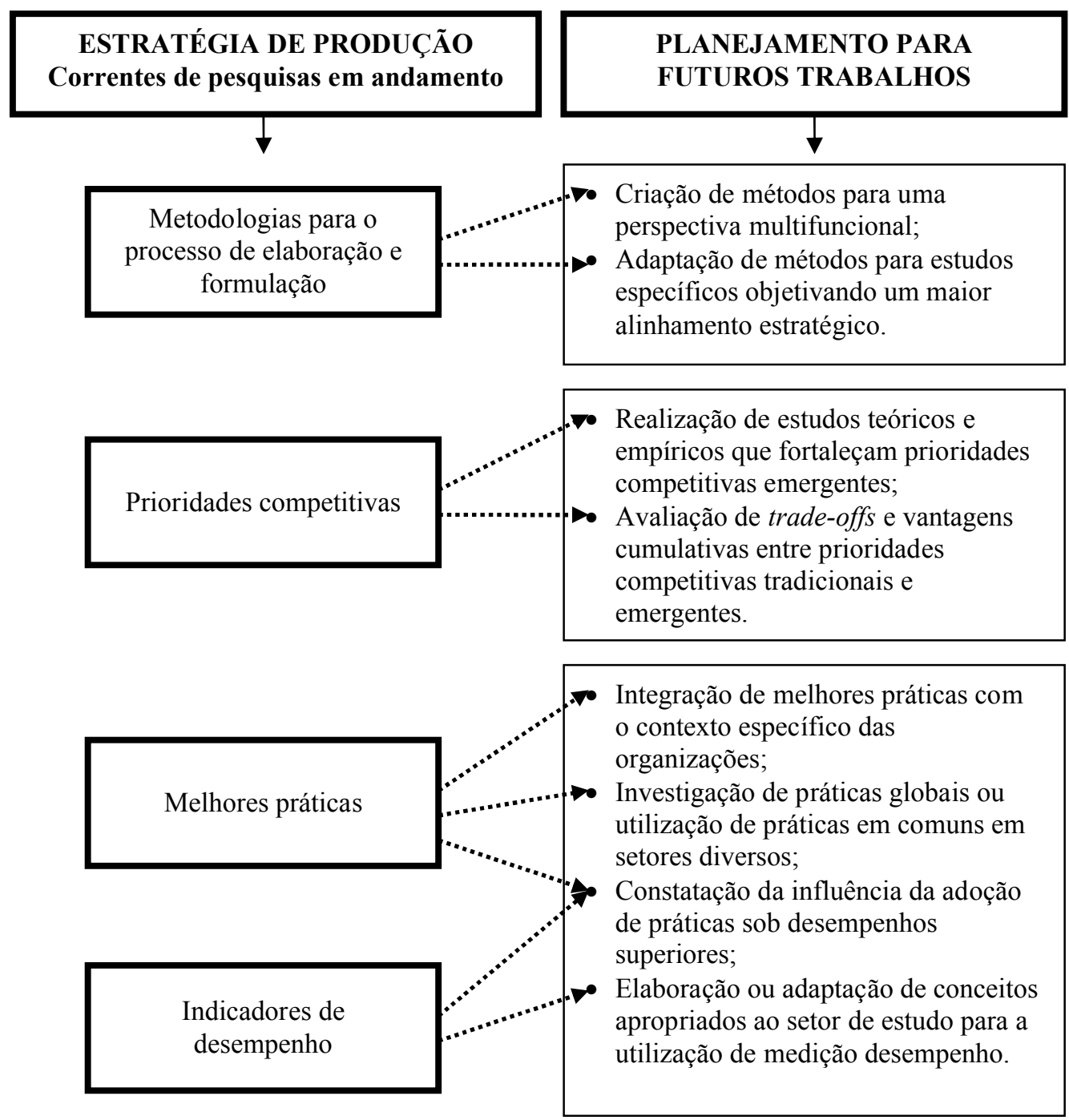

\section{Considerações finais}

Mediante um novo construto que desmembrou a estratégia de produção em seis correntes de pesquisas e por uma ampla revisão, este trabalho identificou lacunas e sugeriu um planejamento para futuras pesquisas na área. Descobriu-se que das seis linhas de pesquisa, quatro estão em andamento: (a) as metodologias para o processo de elaboração e formulação das estratégias, sobretudo, no que diz respeito ao entendimento do alinhamento estratégico; (b) as prioridades competitivas; (c) as melhores práticas; e (d) os indicadores de desempenho.

Então, tornou-se possível refletir sobre os problemas de pesquisa encontrados na literatura, proporcionando um maior esclarecimento dos mesmos. Descobriu-se que alguns conceitos teóricos - modelos de formulação e indicadores de desempenho da manufatura - podem ser aplicados mais efetivamente mediante adaptações para setores específicos. Além disso, foram direcionadas futuras pesquisas para as prioridades competitivas emergentes. Ademais, mostrou-se que o campo de estudo em melhores práticas está em plena expansão apresentando carência de estudos em 
diferentes áreas de decisões da manufatura e, também, em trabalhos que correlacionam o contexto específico e a performance organizacional.

\begin{abstract}
The main aim of this paper is to identify research gaps and to recommend a planning for future studies in the field of manufacturing strategy, mainly those linked to best practices and operational performance. As this is a theme that has been widely discussed for almost half a century, this investigation contributes to present research areas based on a new global and systematic vision and a systematization of literature. A complete bibliographic revision on manufacturing strategy is carried out. Four out of the six research areas identified have a dynamic process of investigation. Finally, both a reflection on the found gaps and suggestions for future studies are presented.
\end{abstract}

Key-words: manufacturing strategy, best practices, operational performance.

\title{
Referências
}

ADAM, E.E.; SWAMIDASS, P.M. Assessing operations management from strategic perspective. Journal of Management, v.15, n.2, p.181-203, 1989.

cross'

ANGELL, L.C.; KLASSEN, R.D. Integrating environmental issues into the mainstream: an agenda for research in operations management. Journal of Operations Management, v.17, p.575-598, 1999.

cross ${ }^{\text {ref }}$

BANKER, R.D.; FIELD, J.M.; SCHROEDER, R.G.; SINHA, K.K. Impact of work teams on manufacturing performance: a longitudinal field study. Academy of Management Journal, v. 39, n.4, p.867-890, 1996.

cross ${ }^{\text {ef }}$

BARNEY, J.B. Firm resources and sustained competitive advantage. Journal of Management, v.17, n.1, p.99-120, 1991. cross ${ }^{\text {ref }}$

BEAUMONT, N. Best Practice in Australian manufacturing sites. Techovation, v. 25, p. 1291-1297, 2005. cross ${ }^{\text {ref }}$

BOYER, K.K.; PAGELL, M. Measurement issues in empirical research: improving measures of operations strategy and advanced manufacturing technology. Journal of Operations Management, v.18, p. 361-374, 2000.

cross ${ }^{\text {ref }}$

BOLDEN, R. et al. A new taxonomy of modern manufacturing practices. International Journal of Operations \& Production Management,v.17, n.11/12, p.1112-1130, 1997.

cross ${ }^{\text {ref }}$

BOZARTH, C.; MCDERMOTT, C. Configurations in manufacturing strategy: a review and directions for future research. Journal of Operations Management, v. 16, n.4, p. 427-439, 1998.

cross ${ }^{\text {ref }}$

BOYER, K.K. Longitudinal linkages between intended and realized operations strategies. International Journal of Operations \& Production Management, v.18, n.4, p. 356-373, 1998.

cross ${ }^{\text {ref }}$

CHRISTIANSEN, T. et al. A mapping of competitive priorities, manufacturing practices, and operational performance in groups of Danish manufacturing companies. International Journal of Operations \& Production Management, v. 23, n.10, p. 1163-1183, 2003.

cross 
CUA, K.O. MCKONE, K.E.; SCHROEDER, R.G. Relationships between implementation of TQM, JIT and TPM and manufacturing performance. Journal of Operations Management, v.19, n.6, p.675-694, 2001.

cross ${ }^{\text {ref }}$

DAVIES, A.J.; KOCHHAR, A.K. Manufacturing best practice and performance studies: a critique. International Journal of Operations \& Production Management, v.22, n.3, p. 289-305, 2002.

cross

FITZ-ENZ, J. The truth about best practices: what they are and how to apply them. Human Resource Management, v. 36, n.1, p. 97-103, 1997.

cross ${ }^{\text {ref }}$

FYNES. B.; VOSS. C.; BÚRCA, S. The impact of supply chain relationship dynamics on manufacturing performance. International Journal of Operations \& Production Management, v.25, n.1, p.6-19, 2005.

cross ${ }^{\text {ref }}$

GARVIN, D.A. Manufacturing strategic planning. California Management Review, v. 35, n. 4, p.85-106, 1993.

GERWIN, D. A agenda for research on the flexibility of manufacturing processes. International Journal \& Production Management, v.7, n.1, p.38-49, 1987.

cross ${ }^{\text {ref }}$

GILLEY, M.K. RASHEED, A. Making more by doing less: an analysis of outsourcing and its effects on firm performance. Journal of Management, v. 26, n.4, p.763-790, 2000.

cross'ref

GRANT, R. The resource-based theory of competitive advantage: implications for strategy formulation. California Management Review, v.33, n.3, p. 114-135, 1991.

HAYES, R.H.; WHEELWRIGHT, S.C. Restoring our competitive edge: competing through manufacturing. New York: John e Wiley, 1984.

HILL, T. Manufacturing strategy: text and cases. $2^{\mathrm{a}}$ ed. EUA: Richard D. Irwin Inc, 1993.

HORTE, S.A.; LINDERBEG, P.; TUNALV, C. Manufacturing strategies in Sweden. International Journal of Production Research, v.25, n.11, p.1573-1586, 1987.

JIMÉNEZ, J.B. de; LORENTE, J.J.C. Environmental performance as an operations objective. International Journal of Operations \& Production Management, v.21, n.12, p.1553-1572, 2001.

cross ${ }^{\text {ref }}$

KLIPPEL, M. ANTUNES JÚNIOR, J.A.V.; PAIVA, E.L. Estratégia de produção em empresas com linhas de produtos diferenciadas: um estudo de caso em uma empresa rodoferroviária. Gestão \& Produção, v.12, n.3, p.417-428, 2005.

cross ${ }^{\text {ref }}$

KOTHA, S.; ORNE, D. Generic manufacturing strategies: a conceptual synthesis. Strategic Management Journal, v.10, n.3, 211-231, 1989.

crossef

KRAJEWSKI, L.J.; RITZMAN, L.P. Operations management: strategy and analysis. EUA: Addison-Wesley Longman, Inc, 2000.

KRAUSE, D.R.; PAGELL, M.; CURKOVIC, S. Toward a measure of competitive priorities for purchasing, Journal of Operations Management, v. 19, n.4, p.497-512, 2001.

cross ${ }^{\text {ref }}$

LAUGEN, T.B.; BOER, N.A.H.; FRICK, J. Best manufacturing practices: what do the best-performing companies do? International Journal of Operations \& Production Management, v.25, n.2, p.131-150, 2005.

crossef

LEONG G.K.; SNYDER, D.L.; WARD, P.T. Research in the process and content of manufacturing strategy. OmegaInternational Journal of Management Science , v.18, n.2: p.109-122, 1990.

cross ${ }^{\text {ref }}$ 
MAIA, J.L.; CERRA, A.L.; ALVES FILHO, A.G. Inter-relações entre estratégia de operações e a gestão da cadeia de suprimentos: estudos de caso no segmento de motores para automóveis. Gestão \& Produção, v.12, n.3, p.377-391, 2005.

MILLS, J.; PLATTS, K.; GREGORY, M. A Framework for the design of manufacturing strategy process: a contingency approach. International Journal of Operations \& Production Management, v.15, n.4. p.17-49, 1995. cross ${ }^{\text {ref }}$

NARASIMHAN, R.; SWINK, M.; KIM, S.W. An exploratory study of manufacturing practice and performance interrelationships: implications for capability progression. International Journal of Operations \& Production Management, v.25, n.10, p.1013-1033, 2005.

cross ${ }^{\text {ef }}$

NOGUEIRA, E.; ALVES FILHO, A.G.; TORKOMIAN A.L.V. Empresas de revestimento cerâmico e suas estratégias competitivas. Gestão \& Produção, São Carlos, v.8, n.1, p.84-99, 2001.

PAIVA, E.L.; FENSTERSEIFER, J.E.; CARVALHO JÚNIOR, J.M. Estratégia de produção e de operações: conceitos, melhores práticas e visão de futuro.Porto Alegre: Bookman, 2004.

PILKINGTON, A. Manufacturing strategy regained: evidence for the demise of best-practice. California Management Review, v. 41, n.1, p. 31-42, 1998.

PIRES, S.R.I. Gestão estratégica da produção. Piracicaba: Unimep, 1995.

PLATTS, K.W. Characteristics of methodologies for manufacturing strategy formulation. Computer Integrated Manufacturing Systems, v.7, n.2, p.93-99, 1994.

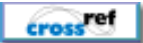

PORTER, M.E. Competitive strategy: techniques for analyzing industries and competitors. New York : Free Press, 1980.

SANTOS, F.C.A. Integration of human resource management and competitive and competitive priorities of manufacturing strategy. International Journal of Operations \& Production Management, v.20, n.5, p.610-628, 2000. cross ${ }^{\text {ref }}$

SCHROEDER, R.G. Operations management: decision making in the operations function. $4^{\text {a }}$ ed. EUA: McGraw-Hill, 1993.

SCHROEDER, R.G.; ANDERSON, J.C.; CLEVELAND.G. The content of manufacturing strategy: An empirical study. Journal of Operations Management, v.6, n.3-4, p.405-415, 1986.

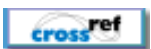

SEMLER, S.W. Systematic agreement: a theory of organizational alignment. Human Resource Development Quarterly, v.8, n.1, p.23-40, 1997.

cross ${ }^{\text {ref }}$

SHAH, R.; WARD; P.T. Lean manufacturing: context, practice bundles, and performance. Journal of Operations Management, v.21, n.2, p.129-149, 2003.

cross ${ }^{\text {ref }}$

SILVA, E. M. da.; SANTOS, F.C.A. Análise do alinhamento da estratégia de produção com a estratégia competitiva na indústria moveleira. Revista Produção, v. 15, n. 2, p. 286-299, 2005.

SKINNER, W. Manufacturing - the missing link in corporate strategy. Harvard Business Review, n.47, n.3, p.136-145, 1969.

SLACK, N. et al. Administração da produção. $2^{a}$ ed. São Paulo: Atlas, 2002.

STEPANOVICH, P.L.; MUELLER, J.D. Mapping strategic consensus. Journal of Business and Management, v.8, n.2, p.147-160, 2002.

STONEBRAKER, P.W.; LEONG G.K. Operations strategy: focusing competitive excellent. Massachusetts: Allyn an Bacon, 1994.

SUN, H.; HONG, C. The alignment between manufacturing and business strategies: its influence on business performance. Technovation, v. 22, n. 11, p. 699-705, 2002. 
SWAMIDASS, P.M.; NEWELL, W.T. Manufacturing strategy, environmental uncertainty and performance: a path analytic model. Management Science, v.33, n.4, p.509-524. Apr, 1987.

cross ref

SWINK, M.; NARASIMHAN, R.; KIM, S.W. Manufacturing practices and strategy integration: effects on cost efficiency, flexibility, and market-based performance. Decision Sciences, v.36, n.3, p.427-457, 2005.

cross ${ }^{\text {ref }}$

UNGAN, M. Factors affecting the adoption of manufacturing best practices. Benchmarking. v.11, n.5, p.504-520, 2004 . cross ${ }^{\text {ref }}$

VACHON, S.;KLASSEN, R.D. Green project partnership in the supply chain: the case of the package printing industry. Journal of Cleaner Production, v.14, n.6-7, p.661-671, 2006.

cross'ref

VICKERY, S.K.; DROGE, C.; MARKLAND, R.E. Production Competence and business strategy: do they affect business perfomance? Decision Sciences, v.24, n.2, p.435-455, 1993.

cross ${ }^{\text {ref }}$

VOSS, A.C. Alternative paradigms for manufacturing strategy. International Journal of Operations \& Production Management, v.15, n.4, p.5-16, 1995.

cross ${ }^{\text {ref }}$

WERNERFELT, B. A resource-based view of the firm. Strategic Management Journal. v.5, n.2, p. 171-180, 1984. cross ${ }^{\text {ref }}$

YOUNDT, M.A.; SNELL, C.A.; DEAN, J.W.; LEPACK, D.P.; Human resource management, manufacturing strategy and firm performance. Academy of Management Journal, v. 39, n.4, p.836-866, 1996.

cross ${ }^{\text {ref }}$

\section{Dados dos autores:}

Nome completo: Eliciane Maria da Silva

Filiação institucional: Universidade de São Paulo, Escola de Engenharia de São Carlos (EESC/USP)

Departamento: Engenharia de Produção

Endereço: Avenida TrabalhadorSãocarlense, 400 - Centro, São Carlos-SP - Brasil - 13566-590

Telefones para contato: (16) 3373-8286 ou (16) 3398-7246

e-mail: eliciane@sc.usp.br

Nome completo: Fernando César Almada Santos

Filiação institucional: Universidade de São Paulo, Escola de Engenharia de São Carlos (EESC/USP)

Departamento: Engenharia de Produção

Função: Professor doutor

Endereço: Avenida TrabalhadorSãocarlense, 400 - Centro, São Carlos-SP - Brasil - 13566-590

Telefones para contato: (16) 3373-9385 ou (16) 3373-9428

e-mail: $\underline{\text { almada@sc.usp.br }}$ 
Recebido para publicação em: 15/10/2006

Aceito para publicação em: 18/02/2007 